\title{
Review
}

Clinical nephron

Practice

\section{Lupus Nephritis: From Pathogenesis to Targets for Biologic Treatment}

\author{
Yujuan Liu Hans-Joachim Anders \\ Nephrologisches Zentrum, Medizinische Klinik und Poliklinik IV, Klinikum der Universität, München, \\ Munich, Germany
}

\section{Key Words}

Immune complex · Belimumab · Interferon · Systemic lupus erythematosus · Abatacept · Proteinuria

\begin{abstract}
Background/Aims: Lupus nephritis is an organ manifestation of systemic autoimmunity. Current treatment algorithms are still based on unselective immunosuppressive drugs. There is hope that highly selective biological drugs could be as or even more effective but less toxic. A profound understanding of the pathogenesis of lupus nephritis is necessary to identify the optimal molecular targets. Methods: PubMed and www.clincialtrials.gov were searched using 'lupus nephritis' as the key word. Results: The pathogenesis of lupus nephritis is based (1) on the mechanisms that lead to loss of tolerance against nuclear autoantigens, i.e. systemic lupus, and then (2) on the mechanisms of immune complexinduced intrarenal inflammation. Systemic lupus develops when genetic variants allow autoimmunization against nuclear autoantigens, e.g. by impairing lymphocyte depletion via apoptosis, opsonization, and rapid phagocytic clearance. This allows endogenous nucleic acids to directly activate Toll-like receptors on dendritic cells or B cells, a process that drives IFN-a-driven immunity, antigen presentation, and the activation of autoreactive lymphocyte subsets. Activation of $B$ cells and their maturation to plasma cells promotes autoantibody production and subsequent immune complex glomerulonephritis. Complement and numerous proinflam-
\end{abstract}

matory cytokines drive the inflammatory process that can cause kidney injury, scarring, and chronic kidney disease. Conclusion: Systemic lupus is more a variable syndrome than a single disorder based on heterogeneous genetic variants and complex aberrant immune alterations. This makes it less likely that a single specific biological drug will be as efficient as currently used unselective immunosuppressive drugs. Autoantibody production and intrarenal immune complex formation are the hallmark of lupus nephritis. However, kidney injury and scarring also result from local amplification of tissue inflammation. Therefore, a combination of unselective immunosuppressive and biological drugs that block immune cell recruitment or proinflammatory cytokines may be promising to improve disease outcomes in lupus nephritis.

๑ 2014 S. Karger AG, Basel

\section{Introduction}

Biological drugs have significantly improved outcomes in many autoimmune disorders, but so far clinical trials have failed to demonstrate any significant benefit of biological drugs in lupus nephritis. This is surpris-

Biologic Treatment in Glomerular Disease D. Jayne, Cambridge

V. Tesar, Prague

\section{KARGER}

E-Mail karger@karger.com

www.karger.com/nec
(C) 2014 S. Karger AG, Basel

$1660-2110 / 14 / 1284-0224 \$ 39.50 / 0$
Hans-Joachim Anders, MD Nephrologisches Zentrum

Medizinische Klinik und Poliklinik IV, Klinikum der Universität München

Ziemssenstrasse 1, DE-80336 Munich (Germany)

E-Mail hjanders@med.uni-muenchen.de 
Table 1. Pathomechanisms in SLE and lupus nephritis

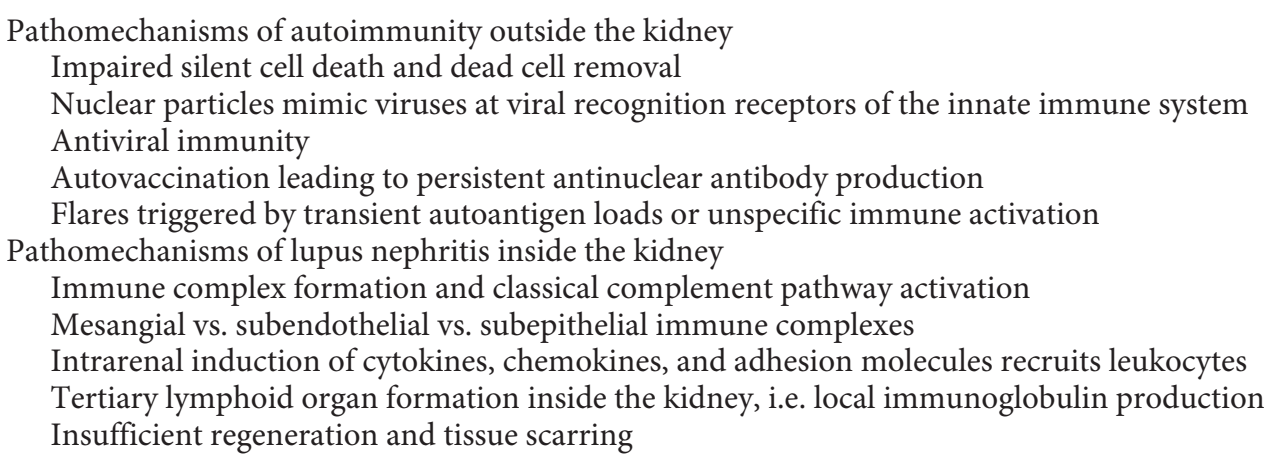

ing because all autoimmune disorders, including systemic lupus erythematosus (SLE), are based on antigen-presenting cells that trigger the activation and proliferation of autoreactive lymphocyte subsets. So what distinguishes the pathogenesis of SLE from other autoimmune disorders? Why do clinical trials often fail to validate drug efficacy previously demonstrated in preclinical experiments or uncontrolled cohort studies? Disease heterogeneity is an important factor that distinguishes SLE from other autoimmune disorders and which remains a challenge for clinical trial design. Genome-wide association studies have documented that numerous different genetic alterations, each of them conferring only a minor risk contribution, are present in SLE patients. This implies that SLE is rather a clinical syndrome that develops from different combinations of genetic alterations that cause systemic autoimmunity through different avenues of immune dysregulation. This may explain why some but not all patients benefit from biological drugs that modulate highly selective targets. Nonetheless, to move beyond unselective immunosuppressive drugs in lupus nephritis management, it is necessary to identify common pathways in the pathogenesis of SLE and lupus nephritis. In this review, we briefly summarize current concepts of disease pathogenesis in SLE and lupus nephritis and present molecular and cellular targets for biological drugs to improve disease outcomes.

\section{Pathogenesis of SLE and Lupus Nephritis}

As we have recently described the current pathogenic concepts of SLE and lupus nephritis in detail elsewhere [1], only a brief summary is provided here (table 1).

\section{Systemic Autoimmunity in SLE}

SLE develops from a loss-of-tolerance for nuclear autoantigens as is evident from the presence of antinuclear antibodies. As tolerance against cell nuclei is normally assured by numerous mechanisms and genes, many different combinations of genetic and environmental factors can break tolerance, cause antinuclear antibody positivity, and eventually trigger SLE. The major checkpoints of immune dysregulation are described in table 1 and below.

Impaired Silent Cell Death and Dead Cell Removal. Genetic variants that impair physiological (immunologically silent) suicide of autoreactive lymphocytes during negative selection in lymphoid organs drive secondary necrosis and the release of nuclear material into the extracellular space. Similarly, this is also the case with environmentally induced tissue cell necrosis, e.g. a sunburn or a trauma increases the amount of extracellular nuclear material. In SLE, often genetic variants that impair the phagocytic clearance of such extracellular nuclear particles keep nuclear particles in the extracellular space [2]. For example, insufficient phagocytic clearance of extracellular neutrophil traps contributes to this phenomenon [3].

Nuclear Particles Trigger Antiviral Immunity via Innate Viral Recognition Receptors. Extracellular nuclear particles share structural and molecular similarities with viral particles and their nucleic acid components can activate dendritic cells and B cells via Toll-like receptors 7 and 9 [4]. This specifically triggers the induction of interferon- $\alpha$ and subsequently a 'pseudo' antiviral immune response, which accounts for unspecific symptoms of SLE including fatigue, fever, myalgia, and arthralgia [5].

Autovaccination Leads to Persistent Antinuclear Antibody Production. Because nuclear autoantigens are pre- 
sented in the context of innate immune activation, induced costimulation promotes the activation of $\mathrm{T}$ and $\mathrm{B}$ cell subsets with specificities for nuclear autoantigens. This process involves $\mathrm{B}$ cell maturation and differentiation into plasma cells that produce antinuclear antibodies. Memory T cells and bone marrow long-lived plasma cells assure lifelong immune memory, which cannot be eradicated by standard therapies conceptually like immune memory obtained by previous vaccinations [6]. Unspecific lymphoproliferation drives lymphadenopathy as a manifestation of SLE.

Incident Cell Necrosis or Unspecific Immune Activation Trigger SLE Flares. Sudden massive cell death (sunburn or trauma) or exposure to immunostimulatory agents (e.g. during infections) mimic antigen re-exposure or provide unspecific stimuli of innate and adaptive immunity that may expand autoreactive lymphocyte clones and cause a SLE flare [7].

Intrarenal Pathomechanisms of SLE-Related Nephritis Immune Complex Formation and Classical Complement Pathway Activation. Lupus nephritis does not develop in the absence of antinuclear antibodies [8]. Circulating polyclonal autoantibodies bind to intrarenal nucleosomes and other autoantigens, which leads to local complement activation, cell injury, and subsequent cytokine and chemokine secretion.

The Immune Complex Formation Site Determines Lupus Nephritis Outcomes. The polyclonal lupus autoantibody isotypes can localize to different compartments within the glomerulus, which affects the type of histopathological lesion as well as the alterations of glomerular function [9]. Immune complex formation in the mesangium induces mesangioproliferative glomerulonephritis (lupus nephritis classes I and II), which is often mild and rarely progresses to end-stage kidney disease. Subendothelial immune complex formation (lupus nephritis classes III and IV) causes vascular obstruction by endothelial cell swelling and clotting, which promotes a decline of glomerular filtration rate. Vascular necrosis and glomerular basement membrane ruptures promote hematuria, crescent formation, and subsequently glomerulosclerosis. Subepithelial immune complex formation (membranous lupus nephritis class V) injures podocytes, which promotes massive proteinuria and podocyte lossrelated glomerulosclerosis.

Induction of Cytokines, Chemokines, and Adhesion Molecules Recruits Leukocytes. Leukocyte recruitment amplifies intrarenal inflammation and promotes secondary tissue injury related to tissue inflammation and drives a vicious cycle of inflammation-induced tissue injury and injury-related inflammation. To target this pathomechanism, anti-inflammatory drugs that do not cause global immunosuppression may be sufficient.

Tertiary Lymphoid Organ Formation inside the Kidney. Local expression of lymphotoxin and homeostatic chemokines drives tertiary lymphoid organ formation at sites of chronic inflammation to promote the (auto-) immune response, e.g. by local autoantibody production.

Insufficient Regeneration and Tissue Scarring. Attempts to heal tissue injury often create new lesions. Lesions of hyperactive repair include hyperproliferation of mesangial cells (mesangioproliferative lupus nephritis), endothelial cells (endocapillary lupus nephritis), and parietal epithelial cells (crescentic lupus nephritis). Lesions of insufficient repair include podocyte loss-related scarring (FSGS and glomerulosclerosis).

\section{Therapeutic Targets for (Biological) Drugs}

The current management of lupus nephritis remains based on steroids, cyclophosphamide, azathioprine, and mycophenolate mofetil, which are all unselective immunosuppressive drugs that suppress multiple components of adaptive immunity [10]. These drugs have proven to be efficient in reducing lupus nephritis disease activity, but the long-term outcomes of lupus nephritis have not further improved during the last 30 years. Unselective immunosuppressive drugs are associated with potentially life-threatening infectious complications. Therefore, it remains an unmet medical need to develop new drugs that more specifically interfere with the pathomechanisms of SLE and cause less side effects [11]. In the following we discuss several targets of interest and provide the rationale to explore them for the treatment of lupus nephritis (fig. 1). Past or ongoing clinical trials with target-related drugs are listed in table 2.

\section{Aberrant Cell Death and Insufficient Clearance of Dead Cells}

Currently no biological drugs are available that specifically control this aspect of lupus pathogenesis. The prevention of sunburns by avoiding UV light exposure and using sunscreen remains important.

\section{Immunostimulatory Effects of Endogenous Nucleic}

Acids and IFN- $\alpha$-Mediated Immunity

Toll-like receptor 7/9 blockade can suppress nucleic acid autoadjuvant-driven disease activity of lupus nephri- 
Table 2. Biological drugs for lupus nephritis listed in www.clinicaltrials.gov

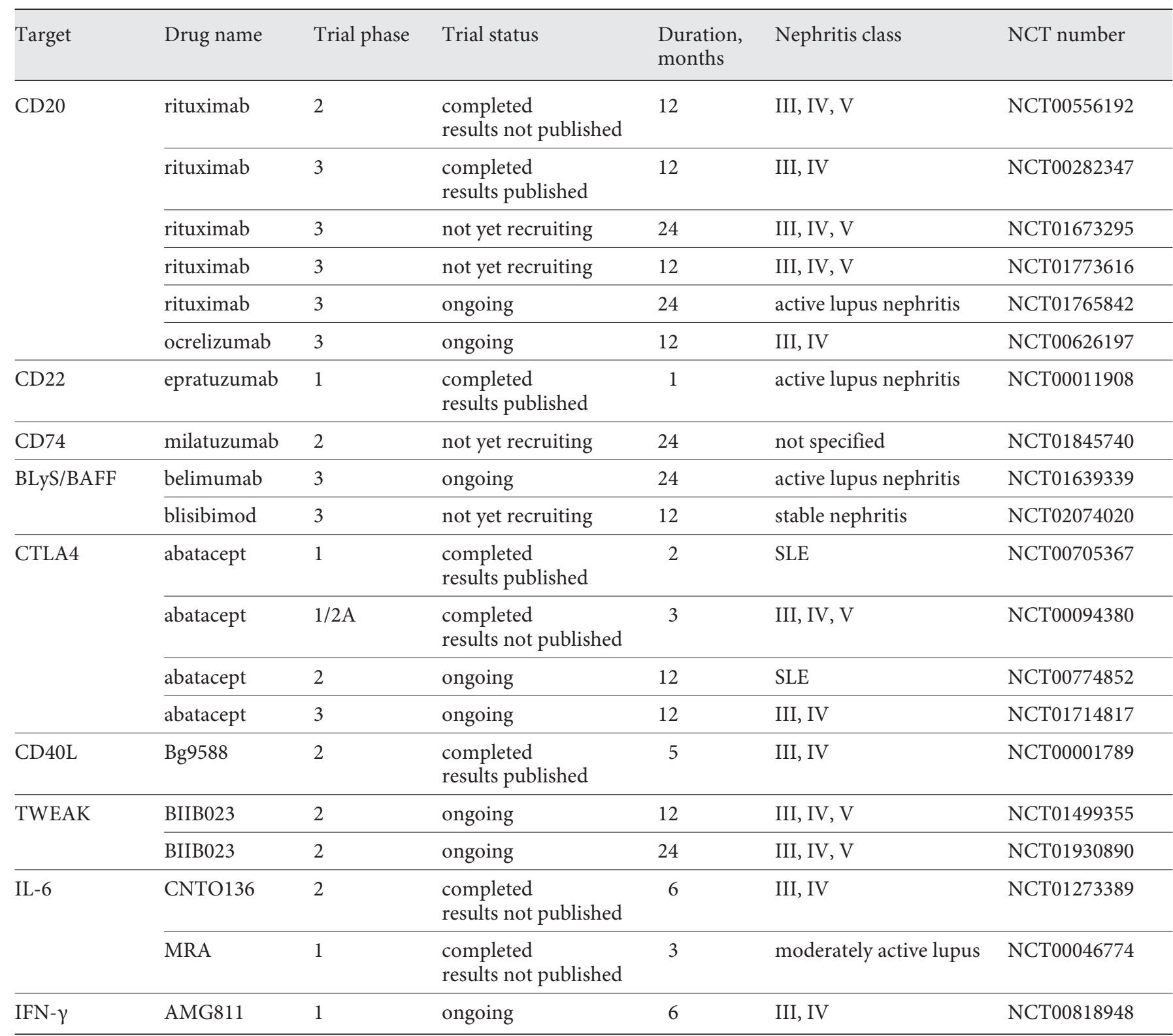

Fig. 1. Pathomechanisms and treatment targets in lupus nephritis. a Genetic variants of homeostatic lymphocyte death and of the rapid clearance of dead cells predispose to necrotic cell death and a persistence of nuclear particles in the extracellular space. No specific treatments are available at present for this aspect of lupus pathogenesis. The avoidance of sun burns and toxin exposure can help to prevent disease flares triggered by incident episodes of massive cell death. b Extracellular nuclear particles containing autoadjuvants like CpG-DNA or immunostimulatory RNA together with other Toll-like receptor ligands released by dead cells activate antigen-presenting cells (dendritic cells and B cells) to pres- ent autoantigens together with costimulatory molecules. This process leads to an immune interpretation of the autoantigen as 'foreign' and triggers an adaptive immune response, e.g. autoantigen-specific T and B cells. Several biological drugs intend to interfere with this pathomechanism. c Circulating autoantibodies bind to their respective autoantigens within the kidney, a process referred to as in situ immune complex formation. This activates complement and other inflammatory mediators that can be targeted with biological drugs to control renal inflammation. IFN- $\gamma$, MHCI, MHCII (major histocompatibility complex), TNF, and BAFF/BLyS.
(For figure see next page.) 
a Dysregulated cell death and dead cell clearance

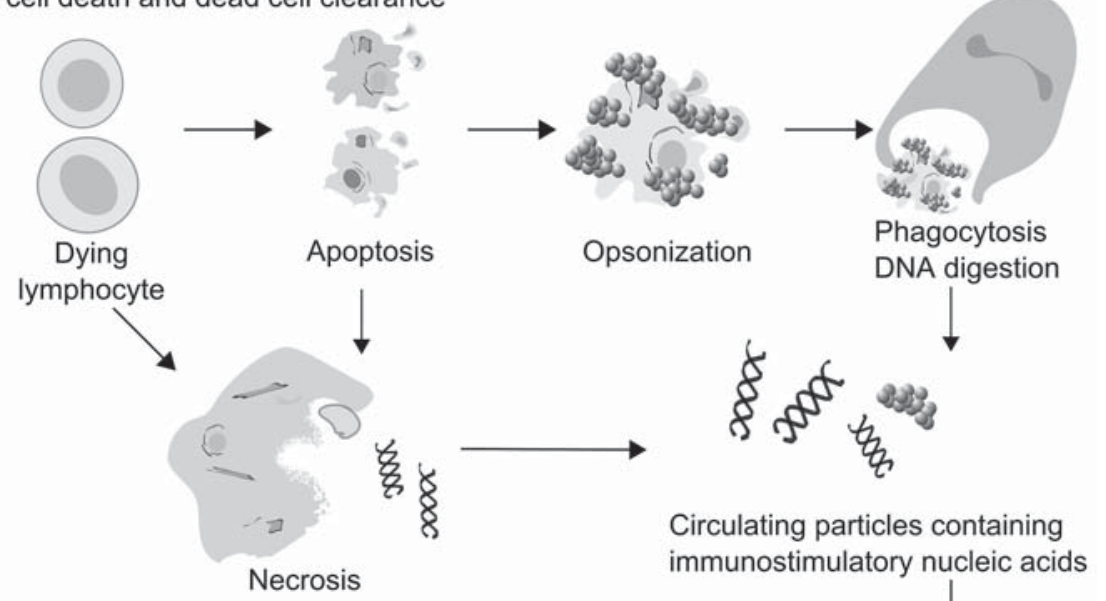

b Auto-vaccination with nuclear antigens

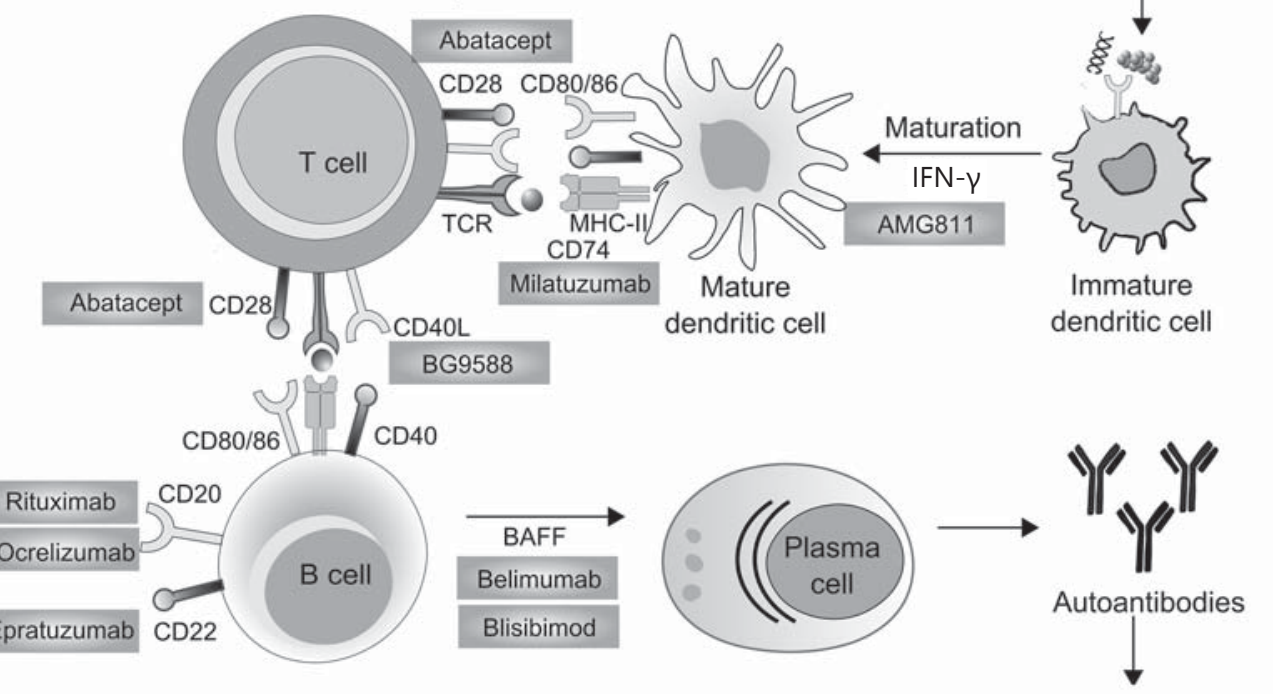

c In situ immune complex formation and glomerulonephritis
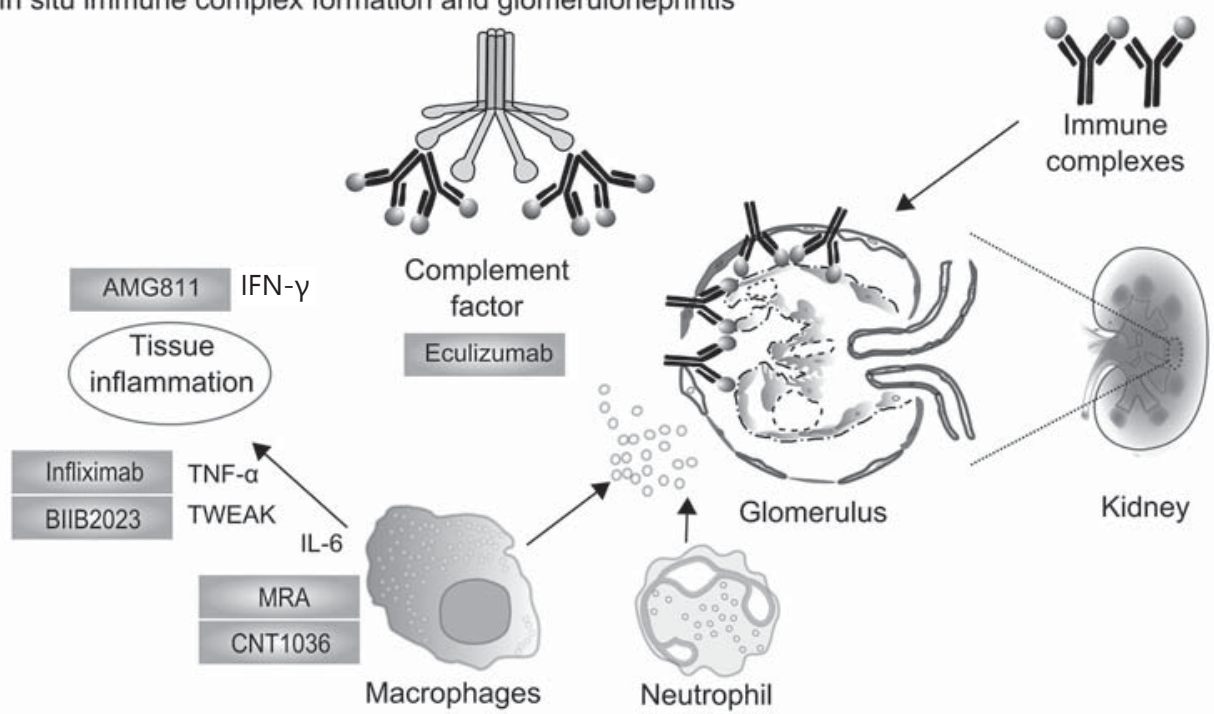
tis $[12,13]$, but the respective antagonists that are to be tested in clinical trials are more based on nucleic acid formats than biological drugs. In contrast, the anti-IFN- $\alpha$ antibody sifalimumab was reported to meet the primary composite endpoint in a phase $2 \mathrm{~b}$ trial of nonrenal lupus patients. Data on its efficacy on lupus nephritis are not yet available.

\section{Autoantigen Presentation and T Cell Activation}

The processing and presentation of autoantigens in the context of costimulation is at the center of every autoimmune disease pathogenesis. Using CTLA-4-Ig to block the interaction between CD80 and CD86 on antigen-presenting cells and CD28 on T cells efficiently suppresses alloimmune $\mathrm{T}$ cell activation after kidney transplantation. However, abatacept failed to succeed to reach the primary endpoint in a randomized trial on the induction phase of lupus nephritis classes III and IV, although abatacept therapy had some effects on plasma levels of dsDNA autoantibodies and complement recovery [14]. CD40 and CD40L also promote costimulation, but three trials with anti-CD40L failed to demonstrate efficacy. Abetimus is a drug that modulates autoimmunity via antigen recognition by $\mathrm{T}$ cells. Abetimus is composed of a series of linked oligonucleotides, which block the binding of anti-dsDNA antibodies to their autoimmune targets and tolerize B cells with antigen-specificity for DNA. Unfortunately, the results in clinical trials have been very modest. The concept of anti-dsDNA-specific therapeutic interventions does not seem very promising as they target only a very small subset of autoreactive B cells in SLE. Antigen presentation requires peptide processing and loading into HLA molecules, a process that involves a nonredundant role for cathepsin $S$, the inhibition of which suppresses IgG autoantibody production and prevents lupus nephritis in animal models [15].

\section{B Cells and Short-Lived Plasma Cells}

B cells clearly contribute to SLE and lupus nephritis, which provides a rationale for the use of drugs that deplete CD20+ B cells and short-lived plasma cells such as rituximab, ocrelizumab, or ofatumumab. Uncontrolled studies on refractory lupus nephritis documented response rates of $75 \%$ [16], but the randomized placebocontrolled LUNAR trial could not demonstrate any benefit of add-on rituximab on top of a profound immunosuppressive regimen for the induction therapy of incident lupus nephritis classes III-V [17]. However, the clinical efficacy of off-label rituximab use by many centers has maintained the interest in B cell ablation in lupus nephri- tis. Based on uncontrolled efficacy data of rituximab monotherapy after methylprednisolone pulsing, the RITUXILUP trial will further test the concept of B cell ablation controlling severe lupus nephritis [18]. CD22 is a $135-\mathrm{kDa} \mathrm{B}$ cell-specific transmembrane sialoglycoprotein that is expressed at the cell surface on mature IgM+ IgD+ B cells, but absent on plasma cells and memory B cells. Blocking CD22 with epratuzumab depletes naïve and transitional $B$ cells via antibody-dependent cellular cytotoxicity, which lowers peripheral B cell counts by $40 \%$. This effect can improve moderate-to-severe nonrenal flares in SLE patients, but efficacy data on lupus nephritis are not yet available [19]. BLyS (B lymphocyte stimulator) is a B cell survival factor that can be blocked with belimumab. Large randomized placebo-controlled trials have proven that add-on belimumab on top of standard maintenance therapy can significantly reduce persistent SLE activity [20], which has led to FDA and EMA approval of belimumab for the maintenance therapy of nonrenal lupus in the USA and Europe. Severe lupus nephritis patients were excluded in the BLISS-56 and BLISS-76 trials, but data from patients with moderate nephritis raise hope that belimumab can also be efficient in severe lupus nephritis [21]. Such a trial is currently recruiting patients. Other B cell-directed biological drugs include the recombinant TACI-human IgG fusion protein atacicept, the BAFF-blocking antibody LY2127399, and a BAFF-receptor antibody [22].

\section{Long-Lived Plasma Cells}

Targeting long-lived plasma cells offers the potential of directly modulating antibody-producing cells that maintain humoral immune memory [6]. These cells reside in survival niches in the bone marrow and in tertiary lymphoid organs within the inflamed tissue. They can be targeted by antithymocyte globulin, small molecule proteasome inhibitors, anti-CD138/CD38 antibodies, or by targeting BLIMP-1. Data from clinical trials that have tested related biological drugs in lupus nephritis are not yet available [6].

\section{Mediators of Tissue Inflammation}

Tissue inflammation involves numerous proinflammatory cytokines, some of which can now be targeted with biological drugs: TNF- $\alpha$ (infliximab), IL-6 (tocilizumab), IL-12 (ustekinumab), IL-17 (ixekizumab and secukinumab), and TWEAK (BIIB023). TNF blockade with infliximab is able to improve severe lupus nephritis, but holds the risk of enhancing autoimmunity and promoting severe infectious complications [23]. The trans- 
continental ATLAS trial that tests TWEAK blockade in the remission phase of lupus nephritis is still ongoing. Macrophage-dependent inflammation is targeted by antimacrophage inhibitory factor IgG, for which a safety study in lupus nephritis patients has been completed. Also the CCL2-CCR2 axis is a promising target to limit macrophage-dependent inflammation. Preclinical experiments suggest that adding a CCL2 inhibitor to a low dose of cyclophosphamide is as efficient as high-dose cyclophosphamide, but avoids myelosuppression and lymphocyte ablation [24]. A first trial documented a positive effect on proteinuria using bindarit as a blocker of this pathway [25].

\section{Summary}

Lupus nephritis develops from extrarenal and intrarenal pathomechanisms. The extrarenal factors include complex combinations of genetic variants in numerous immune pathways that are different in each patient, which may affect success rates of very selective biological drugs in clinical trials. Therefore, treating lupus nephritis with a monotherapy of a selective biological drug should be challenging. Unselective immunosuppressive drugs may remain necessary to suppress the complex interplay of the numerous immune cell subsets that contribute to humoral and cellular autoimmunity in SLE. Lupus nephritis, however, is a classic immune complex glomerulonephritis involving complement-mediated renal inflammation, amplified by infiltrating leukocytes and driven by numerous proinflammatory cytokines. Inflammation-related kidney injury and tissue remodeling cause renal dysfunction and chronic kidney disease in lupus. Therefore, anti-inflammatory biological drugs that limit tissue injury should be attractive combination partners for unselective immunosuppressive drugs that control systemic autoimmunity.

\section{Acknowledgement}

This work was supported by the Deutsche Forschungsgemeinschaft (GRK 1202).

\section{References}

1 Lech M, Anders HJ: The pathogenesis of lupus nephritis. J Am Soc Nephrol 2013;24: 1357-1366.

- Munoz LE, Lauber K, Schiller M, Manfredi AA, Herrmann M: The role of defective clearance of apoptotic cells in systemic autoimmunity. Nat Rev Rheumatol 2010;6:280-289.

-3 Hakkim A, Furnrohr BG, Amann K, Laube B, Abu Abed U, Brinkmann V, Herrmann M, Voll RE, Zychlinsky A: Impairment of neutrophil extracellular trap degradation is associated with lupus nephritis. Proc Natl Acad Sci U S A 2010;107:9813-9818.

4 Marshak-Rothstein A, Rifkin IR: Immunologically active autoantigens: the role of Tolllike receptors in the development of chronic inflammatory disease. Annu Rev Immunol 2007;25:419-441.

5 Migliorini A, Anders HJ: A novel pathogenetic concept-antiviral immunity in lupus nephritis. Nat Rev Nephrol 2012;8:183-189.

-6 Hiepe F, Dorner T, Hauser AE, Hoyer BF, Mei $\mathrm{H}$, Radbruch A: Long-lived autoreactive plasma cells drive persistent autoimmune inflammation. Nat Rev Rheumatol 2011;7:170-178.

7 Allam R, Anders HJ: The role of innate immunity in autoimmune tissue injury. Curr Opin Rheumatol 2008;20:538-544.

8 Lech M, Weidenbusch M, Kulkarni O, Ryu M, Darisipudi MN, Susanti HE, Mittruecker HW, Mak TW, Anders HJ: IRF4 deficiency abrogates lupus nephritis despite enhancing systemic cytokine production. I Am Soc Nephrol 2011;22:1443-1452.

-9 Anders HJ, Fogo AB: Immunopathology of lupus nephritis. Semin Immunopathol 2014.

10 Hahn BH, McMahon MA, Wilkinson A, Wallace WD, Daikh DI, Fitzgerald JD, Karpouzas GA, Merrill JT, Wallace DJ, Yazdany J, Ramsey-Goldman R, Singh K, Khalighi M, Choi SI, Gogia M, Kafaja S, Kamgar M, Lau C, Martin WJ, Parikh S, Peng J, Rastogi A, Chen W, Grossman JM: American College of Rheumatology guidelines for screening, treatment, and management of lupus nephritis. Arthritis Care Res (Hoboken) 2012;64:797-808.

11 Aringer M, Burkhardt H, Burmester GR, Fischer-Betz R, Fleck M, Graninger W, Hiepe F, Jacobi AM, Kotter I, Lakomek HJ, Lorenz HM, Manger B, Schett G, Schmidt RE, Schneider M, Schulze-Koops H, Smolen JS, Specker C, Stoll T, Strangfeld A, Tony HP, Villiger PM, Voll R, Witte T, Dorner T: Current state of evidence on 'off label' therapeutic options for systemic lupus erythematosus, including biological immunosuppressive agents, in Germany, Austria, and Switzerland - a consensus report. Lupus 2012;21:386-401.

12 Guiducci C, Gong M, Xu Z, Gill M, Chaussabel D, Meeker T, Chan JH, Wright T, Punaro M, Bolland S, Soumelis V, Banchereau J, Coffman RL, Pascual V, Barrat FJ: TLR recognition of self nucleic acids hampers glucocorticoid activity in lupus. Nature 2010;465:937-941.
-13 Pawar RD, Ramanjaneyulu A, Kulkarni OP, Lech M, Segerer S, Anders HJ: Inhibition of Toll-like receptor-7 (TLR-7) or TLR-7 plus TLR-9 attenuates glomerulonephritis and lung injury in experimental lupus. J Am Soc Nephrol 2007;18:1721-1731.

14 Furie R, Nicholls K, Cheng TT, Houssiau F, Burgos-Vargas R, Chen SL, Hillson JL, Meadows-Shropshire S, Kinaszczuk M, Merrill JT: Efficacy and safety of abatacept in lupus nephritis: a twelve-month, randomized, doubleblind study. Arthritis Rheumatol 2014;66:379389.

15 Rupanagudi KV, Kulkarni OP, Lichtnekert J, Darisipudi MN, Mulay SR, Schott B, Gruner S, Haap W, Hartmann G, Anders HJ: Cathep$\sin \mathrm{S}$ inhibition suppresses systemic lupus erythematosus and lupus nephritis because cathepsin S is essential for MHC class II-mediated CD4 $\mathrm{T}$ cell and B cell priming. Ann Rheum Dis 2013, Epub ahead of print.

-16 Weidenbusch M, Rommele C, Schrottle A, Anders HJ: Beyond the LUNAR trial. Efficacy of rituximab in refractory lupus nephritis. Nephrol Dial Transplant 2012;28:108-111.

-17 Rovin BH, Furie R, Latinis K, Looney RJ, Fervenza FC, Sanchez-Guerrero J, Maciuca R, Zhang D, Garg JP, Brunetta P, Appel G: Efficacy and safety of rituximab in patients with active proliferative lupus nephritis: the Lupus $\mathrm{Ne}$ phritis Assessment with Rituximab (LUNAR) study. Arthritis Rheum 2012;64:1215-1226. 
18 Condon MB, Ashby D, Pepper RJ, Cook HT, Levy JB, Griffith M, Cairns TD, Lightstone L: Prospective observational singlecentre cohort study to evaluate the effectiveness of treating lupus nephritis with rituximab and mycophenolate mofetil but no oral steroids. Ann Rheum Dis 2013;72: 1280-1286.

19 Wallace DJ, Kalunian K, Petri MA, Strand V, Houssiau FA, Pike M, Kilgallen B, Bongardt S, Barry A, Kelley L, Gordon C: Efficacy and safety of epratuzumab in patients with moderate/ severe active systemic lupus erythematosus: results from EMBLEM, a phase IIb, randomised, double-blind, placebo-controlled, multicentre study. Ann Rheum Dis 2014;73: 183-190.
20 Navarra SV, Guzman RM, Gallacher AE, Hall S, Levy RA, Jimenez RE, Li EK, Thomas M, Kim HY, Leon MG, Tanasescu C, Nasonov E, Lan JL, Pineda L, Zhong ZJ, Freimuth W, Petri MA: Efficacy and safety of belimumab in patients with active systemic lupus erythematosus: a randomised, placebo-controlled, phase 3 trial. Lancet 2011;377:721-731.

21 Manzi S, Sanchez-Guerrero J, Merrill JT, Furie R, Gladman D, Navarra SV, Ginzler EM, D'Cruz DP, Doria A, Cooper S, Zhong ZJ, Hough D, Freimuth W, Petri MA: Effects of belimumab, a B lymphocyte stimulator-specific inhibitor, on disease activity across multiple organ domains in patients with systemic lupus erythematosus: combined results from two phase III trials. Ann Rheum Dis 2012;71: 1833-1838.

22 Gregersen JW, Jayne DR: B-cell depletion in the treatment of lupus nephritis. Nat Rev Nephrol 2012;8:505-514.
3 Aringer M, Houssiau F, Gordon C, Graninger WB, Voll RE, Rath E, Steiner G, Smolen JS: Adverse events and efficacy of TNF-alpha blockade with infliximab in patients with systemic lupus erythematosus: long-term followup of 13 patients. Rheumatology (Oxford) 2009;48:1451-1454.

24 Kulkarni O, Eulberg D, Selve N, Zollner S, Allam R, Pawar RD, Pfeiffer S, Segerer S, Klussmann S, Anders HJ: Anti-Ccl2 Spiegelmer permits $75 \%$ dose reduction of cyclophosphamide to control diffuse proliferative lupus nephritis and pneumonitis in MRL-Fas(lpr) mice. J Pharmacol Exp Ther 2009;328:371-377.

25 Ble A, Mosca M, Di Loreto G, Guglielmotti A, Biondi G, Bombardieri S, Remuzzi G, Ruggenenti P: Antiproteinuric effect of chemokine $\mathrm{C}-\mathrm{C}$ motif ligand 2 inhibition in subjects with acute proliferative lupus nephritis. Am J Nephrol 2011;34:367-372. 